\title{
Nature of the excited states of layered systems and molecular excimers: Exciplex states and their dependence on structure
}

\author{
Heissenbuttel, Marie-Christin; Marauhn, Philipp; Deilmann, Thorsten; Kruger, Peter; Rohlfing, Michael
}

Published in:

Physical Review B

Link to article, DOI:

10.1103/PhysRevB.99.035425

Publication date:

2019

Document Version

Publisher's PDF, also known as Version of record

Link back to DTU Orbit

Citation (APA):

Heissenbuttel, M-C., Marauhn, P., Deilmann, T., Kruger, P., \& Rohlfing, M. (2019). Nature of the excited states of layered systems and molecular excimers: Exciplex states and their dependence on structure. Physical Review $B$, 99(3), [035425]. https://doi.org/10.1103/PhysRevB.99.035425

\section{General rights}

Copyright and moral rights for the publications made accessible in the public portal are retained by the authors and/or other copyright owners and it is a condition of accessing publications that users recognise and abide by the legal requirements associated with these rights.

- Users may download and print one copy of any publication from the public portal for the purpose of private study or research.

- You may not further distribute the material or use it for any profit-making activity or commercial gain

- You may freely distribute the URL identifying the publication in the public portal 


\title{
Nature of the excited states of layered systems and molecular excimers: Exciplex states and their dependence on structure
}

\author{
Marie-Christin Heissenbüttel, ${ }^{1}$ Philipp Marauhn, ${ }^{1}$ Thorsten Deilmann, ${ }^{2}$ Peter Krüger, ${ }^{1}$ and Michael Rohlfing ${ }^{1, *}$ \\ ${ }^{1}$ Institut für Festkörpertheorie, Westfälische Wilhelms-Universität Münster, 48149 Münster, Germany \\ ${ }^{2}$ Center for Atomic-Scale Materials Design (CAMD), Department of Physics, Technical University of Denmark, \\ DK-2800 Kongens Lyngby, Denmark
}

(Received 30 October 2018; published 15 January 2019)

\begin{abstract}
Weakly bound systems, like noble-gas dimers or two-dimensional layered materials (graphite, hexagonal boron nitride, or transition-metal dichalcogenides), exhibit excited electronic states of a particular nature. These so-called exciplex states combine on-site (or intralayer) and charge-transfer (or interlayer) configurations in a well-balanced way. We show by ab initio many-body perturbation theory that the energy and composition of the exciplex states depend sensitively on the bond length or interlayer distance of the material. When the constituents approach each other, the charge-transfer contribution increases and the excitation is redshifted to lower energy. If the system is excited into the exciplex state, then a covalent-like bond results. In consequence, noble-gas dimers form excimer complexes, while layered materials exhibit interlayer contraction.
\end{abstract}

DOI: 10.1103/PhysRevB.99.035425

\section{INTRODUCTION}

Electronic excitations shared by weakly bound components constitute a particular class of quantum-mechanical states [1-4]. Depending on the details of the system, they are known as excimers (forming a short-lived bond between two inert atoms), charge-transfer excitations (between an electron donor and acceptor component), or generally speaking as "exciplex states." The main difference to an on-site excitation is the distinctly nonlocal nature of the excitation, i.e., a strong contribution of electronic configurations in which the excited hole and excited electron are spatially separated. Such excitations can be regarded as entangled states of on-site and charge-transfer contributions.

Exciplex states have been studied for several decades already but are still attracting attention due to their high significance in light-matter interaction [1-4]. They not only exist in pairs of atoms or in pairs of molecules but also can occur whenever two or more components are only weakly bound to each other, e.g., only by van der Waals (vdW) interaction. As we discuss in this paper, this includes the common excitons in layered materials, like graphite, hexagonal boron nitride ( $h$ $\mathrm{BN}$ ), transition-metal dichalcogenides (TMDCs), and others [5-17], which are in the focus of current research. In combination with the peculiar two-dimensional (2D) electronic structure, optical excitations of these systems offer a wide range of interesting effects and potential application.

An exciplex state between two objects (e.g., two atoms or two TMDC monolayers) involves electron-hole excitations on both objects (plus charge-transfer excitations between the two), forming a quantum-mechanical superposition. It should be noted that the resulting exciplex state still involves one excited hole and one excited electron only. This is distinctly

*michael.rohlfing@uni-muenster.de different from biexcitons or other double excitations, which would involve two holes and two electrons, and which are not considered in this work.

The key issue to be discussed in this paper is the relationship between geometrical structure and the excitation energy of charge-neutral electronic excitations. As common in condensed matter, the excited electronic states have potentialenergy surfaces (PES) different from that of the ground state. Here we focus on two complementary types of such relationship, corresponding to two different types of groundstate energetics: covalent bonding and weak interaction. In covalently bonded materials excitation energies commonly decrease when the structure is stretched. Prototypical examples are conventional semiconductors, covalently bonded molecules like $\mathrm{H}_{2}$, and the intralayer physics of TMDCs. In contrast, exciplex states in weakly bound materials (e.g., van der Waals bonded systems) tend to decrease in energy when the structure is compressed. In excimer systems, this effect generates significant bonding in the excited state. In this paper we demonstrate that such behavior is found not only in molecular systems but also equally well (although somewhat weaker) in the common excitons in layered materials. The latter combine strong lateral bonding with weak interlayer binding; consequently, the excitations decrease in energy when the systems are laterally stretched or vertically compressed. We demonstrate that the latter effect results from the exciplex nature of the excitations.

All these issues require a computational approach to excited states in which structural dependence, quantummechanical interaction between orbitals on the various components, exchange and correlation effects among the excited electrons and holes, and dielectric screening effects (both within one component and also between the various components) are all treated on equal footing. Here we employ $a b$ initio many-body perturbation theory (MBPT) [18,19], notably the Bethe-Salpeter equation (BSE) on the basis of a 
preceding band-structure calculation within the common $G W$ approximation (GWA) or (for reasons of numerical efficiency) our simplified LDA $+G d W$ version starting from the localdensity approximation (LDA) [20]. The starting point for MBPT is given by density-functional theory (DFT) for the electronic ground state.

In this paper we focus on homogeneous systems constructed from one type of component only, e.g., the $\mathrm{Ar}_{2}$ dimer, bulk $h$-BN, or bulk TMDCs, in order to illustrate the fundamental principles of exciplex formation from the competition between on-site and charge-transfer configurations. The description of heterogeneous systems, e.g., dimers from two different noble-gas atoms or TMDC heterostructures, would be fully analogous.

The paper is organized as follows. Section II briefly summarizes the theoretical approach relevant for our present work. In Sec. III we discuss electronic excitations of small molecules, both covalently bonded and van der Waals bound, in order to identify exciplex states and to provide the framework of the discussion of excited states of layered systems. Section IV discusses excitations of layered materials in light of the effects found for the molecules and explains the analogies between molecules and layered systems. We suggest possible effects on the structure of layered systems when materials are illuminated with intense light.

\section{THEORETICAL FRAMEWORK}

Here we briefly outline the theoretical framework for describing electronic ground and excited states $[18,19]$. Details (in particular on the excited-state methodology) can be found in Refs. [20-27].

For the electronic ground state we employ DFT, mostly within the LDA. This procedure yields an excellent description of the total-energy landscape (equilibrium geometry, forces, and vibrations) of covalent bonds. For the case of weakly bound van der Waals systems, DFT-LDA tends to overbind, yielding too-short equilibrium distances, too-strong binding energy, and too-high vibrational frequencies. As will be shown below for the example of the $\mathrm{Ar}_{2}$ dimer, this does not significantly falsify the properties of excimer bonds formed by electronic excitation, i.e., DFT still constitutes a good starting point. In cases like graphite or bulk hexagonal BN ( $h$-BN) the ground-state energetics can equally well be deduced from the experimentally known elastic constants (see Sec. IV E).

Excited electronic states are described by MBPT, commonly evaluated within the $G W$ approximation (GWA) for the electron self-energy operator, $\Sigma=i G W$, with $G$ being the single-particle Green function and $W$ the screened Coulomb interaction. Within the commonly employed quasiparticle approximation, charge-neutral excited states (with total momentum close to zero) are expanded as

$$
|S\rangle=\sum_{v c \mathbf{k}} A_{v c \mathbf{k}}^{(S)}|v \mathbf{k} \rightarrow c \mathbf{k}\rangle,
$$

where $|v \mathbf{k} \rightarrow c \mathbf{k}\rangle$ denotes a vertical interband transition between an occupied band $v$ and an empty band $c$. We employ lateral k-point grids of $20 \times 20$ for the TMDC materials, $12 \times 12$ for $h$-BN, and $40 \times 40$ for graphite. In the vertical direction we employ $k_{\perp}=0$ for a TMDC monolayer and four $k_{\perp}$ values for the bulk materials, i.e., up to $6400 \mathrm{k}$-points in the case of graphite. In the case of molecular systems wave-vector expansion in $\mathbf{k}$ does not occur.

The expansion coefficients $A_{v c \mathbf{k}}^{(S)}$ as well as the excitation energy $\Omega_{S}$ of state $S$ are given by the solution of the BSE, within the common approximations. We note that for the layered materials to be discussed in Sec. IV, we employ our simplified LDA $+G d W$ version of MBPT due to its advantageous numerical efficiency [27]. All conclusions hold for GWA and LDA $+G d W$, since both include the physical mechanisms to be discussed in this paper.

In order to obtain total energies of an excited state $S$ we proceed as follows: For each structural configuration (i.e., bond length, lattice constant, etc.) we calculate the excitation energy $\Omega_{S}$ within the BSE. Since the BSE corresponds to the physics of the two-particle Green function, $\Omega_{S}$ denotes the total-energy difference between the ground and excited states and can simply be added to the ground-state total energy (at each structural configuration) [21,28-30]. The groundstate total energy can in principle be obtained within MBPT from the self-consistent single-particle Green function, but this is very difficult due to fundamental and practical problems. Instead, any approach which yields reliable interatomic equilibrium distances and elastic properties can be employed. Here we use DFT-LDA for the energetics of covalent bonds (e.g., the $\mathrm{H}_{2}$ molecule and the intralayer energetics of $h$-BN, graphite, and TMDC), as well as experimental distances and elastic constants for the interlayer energetics of $h$-BN and graphite. As an alternative, exact-exchange (EXX) energy combined with the random-phase approximation (RPA) correlation energy (EXX-RPA) [31], DFT combined with semiempirical dispersion forces [32,33], or similar approaches could be employed. They would, however, not yield any additional insight beyond the discussion in our paper.

\section{MOLECULAR EXCITATIONS}

Before discussing layered materials, we first investigate small molecular systems to provide the basis for analyzing analogies. In addition to general considerations, this demonstrates the validity of our numerical approaches to excitations in both confined and extended systems.

We focus on two prototypical situations (see Fig. 1). Figures 1(a) and 1(b) illustrate a dimer formed from two open-shell objects (labeled A and B), e.g., a $\mathrm{H}_{2}$ molecule formed from two $\mathrm{H}$ atoms. Figures 1(c) and 1(d) illustrate a dimer formed from two closed-shell objects, e.g., a noble-gas dimer formed from two noble-gas atoms. As will be discussed further below, the interaction within a two-dimensional monolayer resembles Figs. 1(a) and 1(b), while the interaction between two 2D monolayers resembles Figs. 1(c) and 1(d).

\section{A. Excitations of a covalently bonded diatomic molecule}

In simplified notation, an open-shell radical has an unpaired electron in its highest occupied orbital. Two such open-shell radicals can construct one bonding molecular orbital (MO), i.e., of $\sigma$ character, and one antibonding MO (of $\sigma^{*}$ character) from their half-filled frontier orbitals, as 

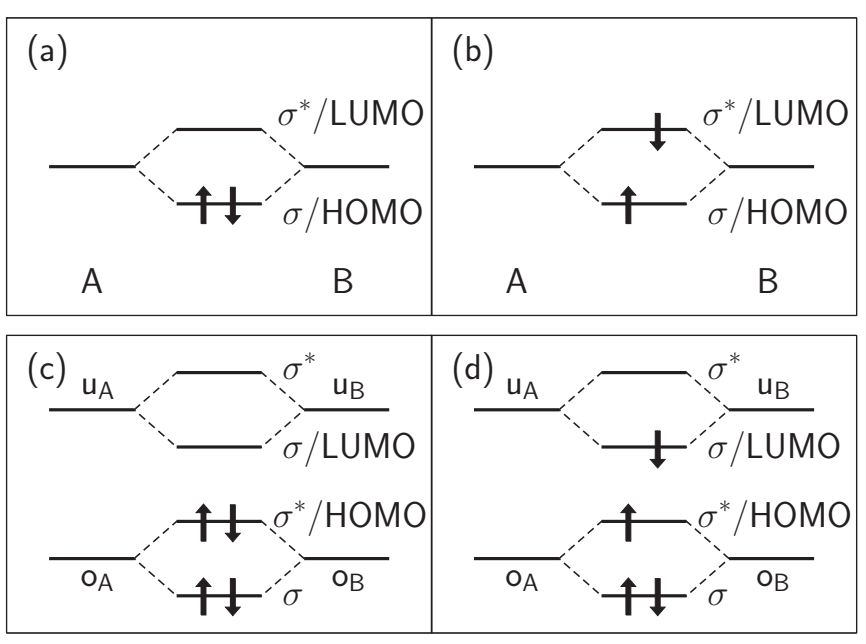

FIG. 1. (a) Electronic ground state of two radicals (A and B), which form a doubly occupied bonding orbital $(\sigma)$. (b) Excited electronic state of (a). Depopulation of the bonding $\sigma$ HOMO and population of the antibonding $\sigma^{*}$ LUMO weakens the bond and causes repulsion of the two constituents. (c) Electronic ground state of two chemically saturated objects (A and B). Each object has a doubly occupied electronic level (o), from which a bonding and an antibonding molecular orbital ( $\sigma$ and $\sigma^{*}$ ) are formed. The double occupation of both $\sigma$ and $\sigma^{*}$ does not constitute any significant covalent bond. The sketch includes unoccupied higher-energy levels (u) on both A and B, which also hybridize into a bonding $(\sigma)$ and an antibonding $\left(\sigma^{*}\right)$ MO. (d) Excited electronic state of (c). Depopulation of the antibonding $\sigma^{*} \mathrm{HOMO}$ and population of the higherenergy bonding $\sigma$ LUMO constitutes twofold "covalent" bonding, causing attraction and bonding of the formerly weakly interacting objects into an excimer.

schematically shown in Fig. 1(a). By occupying the bonding MO with two electrons (in a spin-singlet configuration) a covalent bond is formed. The $\sigma \mathrm{MO}$ then constitutes the highest occupied molecular orbital (HOMO) state, while the $\sigma^{*} \mathrm{MO}$ constitutes the lowest unoccupied molecular orbital (LUMO) state of the molecule. A HOMO-LUMO excitation [see Fig. 1(b)] would then reduce the occupation of the bonding HOMO $(\sigma)$ and occupy the antibonding LUMO $\left(\sigma^{*}\right)$ instead, which in summary weakens the bond. As a consequence, breaking the covalent bond by the HOMO-LUMO excitation will generate a repulsive force between the two components, i.e., excitations tend to increase bond lengths of covalently bonded matter (not to be confused with thermal lattice expansion from dissipation of electronic excitations into anharmonic phonons).

A prototypical case is the $\mathrm{H}_{2}$ molecule. Figure 2 shows its total energy as a function of interatomic distance, for the electronic ground state (from DFT-LDA) and for the lowest spin-triplet and lowest spin-singlet excited states (from a BSE calculation). As one can see, excitation of the $\mathrm{H}_{2}$ molecule generates a PES which is repulsive near the ground-state equilibrium (at $d=0.764 \AA$ in our data, in good agreement with, e.g., $d=0.762 \AA$ found by Gunnarson and Johansson [34]). The excited-state total-energy curves exhibit negative slope $\partial E_{\text {tot }}^{*} / \partial d<0$ (resulting from $\partial \Omega_{S} / \partial d<0$ ). Depending on the excitation, the excited state may yield another equilibrium at enlarged bond length $\left(d=1.21 \AA\right.$ for $\left.{ }^{1} \Sigma_{u}^{+}\right)$or no

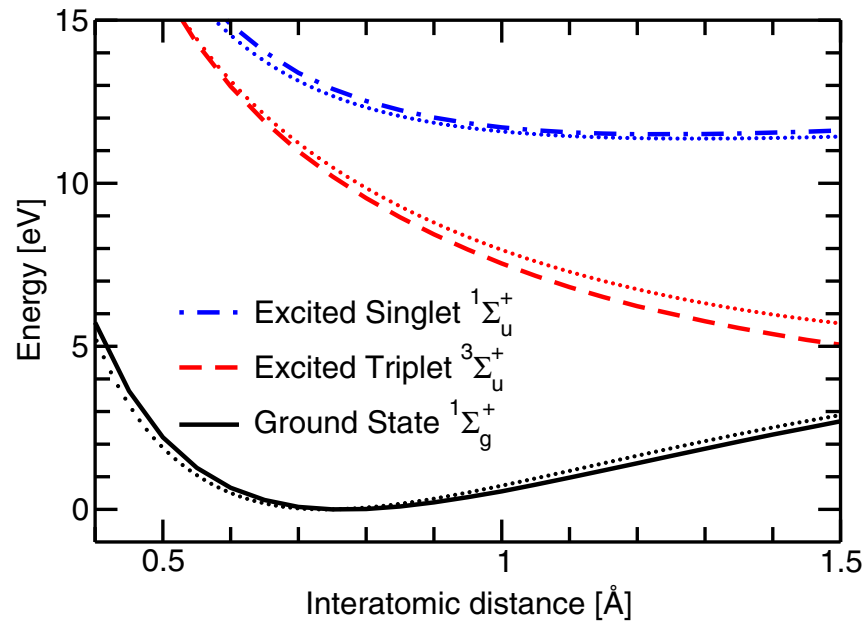

FIG. 2. Total energy of the $\mathrm{H}_{2}$ molecule as a function of the interatomic distance. The ground-state data are taken from DFT-LDA calculations, which yields an equilibrium distance of $0.764 \AA$ in good agreement with the experimental value of $0.741 \AA$ [35]. Among the excited states we focus on the lowest-energy spin-triplet and spinsinglet excitation (mostly HOMO $\rightarrow$ LUMO) and their respective total energies result from adding the $G W / \mathrm{BSE}$ excitation energy to the DFT-LDA ground-state energy at each interatomic distance. For the singlet state ${ }^{1} \Sigma_{u}^{+}$the equilibrium at $d \sim 1.21 \AA(11.5 \mathrm{eV}$ above the ground state) agrees well with measured data of $d=$ $1.29 \AA$ at $E=11.4 \mathrm{eV}$ [35]. The underlying thin dotted lines show data from highly accurate quantum-chemical calculations [36-38].

equilibrium at all (for ${ }^{3} \Sigma_{u}^{+}$) [35]. The curvature near the minima of the PES of Fig. $2\left(34 \mathrm{eV} / \AA^{2}\right.$ for the ground state at $d=$ $0.764 \AA$ and $\sim 5 \mathrm{eV} / \AA^{2}$ for the excited singlet at $d=1.21 \AA$ ) yields vibrational frequencies of $530 \mathrm{meV}$ and $\sim 200 \mathrm{meV}$, as compared to experimental values of 546 and $170 \mathrm{meV}$ [35]. Molecular systems are also studied intensively by highly accurate quantum-chemical approaches, e.g., configuration interaction (CI), or can (for small systems) be treated by variational wave functions to any desired degree of accuracy. Representative results for $\mathrm{H}_{2}$ from the literature [36-38] are included in Fig. 2. The good agreement of our data with experiment and with the quantum-chemical reference data (with slight deviations for the triplet state) clearly supports our conceptual and computational approach.

\section{B. Excitations of a van der Waals bound dimer}

In contrast to the bond between two open-shell systems (see above), two closed-shell systems show distinctly different interaction [see Figs. 1(c) and 1(d)]. Here the starting point is a highest occupied orbital (o) on each of the two systems (labeled A and B), often in a spin-singlet configuration each. Hybridization due to spatial overlap of A and B yields two molecular orbitals of bonding and antibonding character $(\sigma$ and $\sigma^{*}$ ) which are both doubly occupied [see Fig. 1(c)]. In the prototypical case of the $\mathrm{Ar}_{2}$ dimer discussed below the antibonding configuration $\left(\sigma^{*}\right)$ is higher in energy and constitutes the HOMO state of the molecular complex. Since the two $\sigma$ and $\sigma^{*}$ states formed from $o_{\mathrm{A}}$ and $o_{\mathrm{B}}$ are both doubly occupied, a chemical or covalent bond in the sense of 


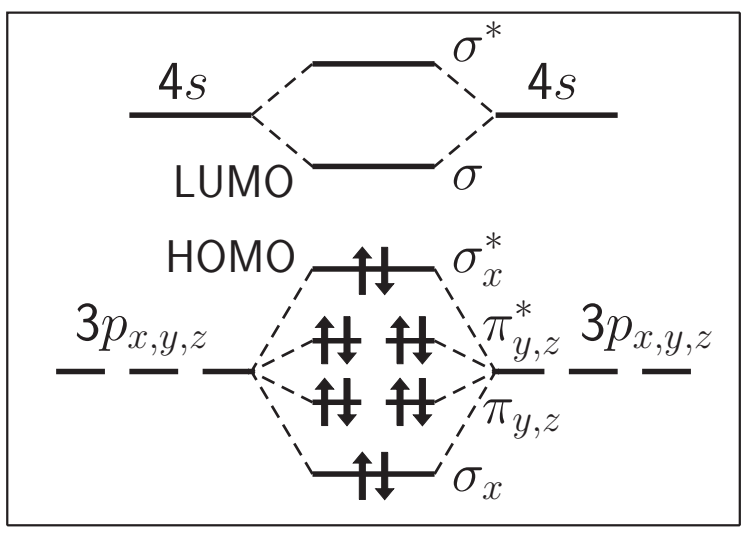

FIG. 3. MO diagram of a dimer of two Ar atoms. The $3 p_{x}$ (with $x$ referring to the dimer axis) orbitals show much stronger hybridization (into $\sigma_{x}$ and $\sigma_{x}^{*}$ ) than $3 p_{y}$ and $3 p_{z}$ (into $\pi_{y, z}$ and $\pi_{y, z}^{*}$ ). Analysis of our computed spatial structure of the orbitals confirms the antibonding nature of the HOMO $\left(\sigma_{x}^{*}\right)$ and the bonding nature of the LUMO $(\sigma)$, in analogy to Fig. 1(c).

Fig. 1(a) is not formed. Instead, such systems are often bound by weak van der Waals interaction, which is not relevant for our present discussion. Note that a similar hybridization also occurs among the lowest unoccupied orbitals (u) of both A and $\mathrm{B}$, also forming a bonding and an antibonding molecular orbital $\left(\sigma\right.$ and $\left.\sigma^{*}\right)$. In the $\mathrm{Ar}_{2}$ dimer the bonding configuration $(\sigma)$ is lower in energy and constitutes the LUMO state of the molecular complex (see below).

Figure 3 shows the schematic MO diagram for the $\mathrm{Ar}_{2}$ dimer, which we find confirmed by our calculations. Obviously, the $\mathrm{Ar}_{2}$ dimer belongs to the system class as illustrated in Fig. 1(c) (except for the additional $\pi$ orbitals, which are not relevant for the following discussion).

In such a weakly bound molecular complex, an electronic HOMO-LUMO excitation (from the antibonding occupied $\sigma^{*}$ HOMO to the bonding unoccupied $\sigma$ LUMO) drastically changes the binding physics between $\mathrm{A}$ and $\mathrm{B}$. The excitation reduces the occupation of the HOMO, such that among the occupied states the remaining double occupation of the occupied bonding $\sigma$ MO dominates, generating a covalent bond while the system is in its excited state. This effect is further enhanced by the occupation of the LUMO, which is also of bonding nature. Simply speaking, as long as the excitation persists there are three electrons in bonding $\sigma$ orbitals and only one electron in an antibonding $\sigma^{*}$ orbital. As a result, the two components form a "covalently" bonded dimer in the excited state, i.e., an excimer or, for a more complex situation or for more than two components, an exciplex structure.

Figure 4 shows the total energy of the $\mathrm{Ar}_{2}$ dimer in the two lowest exciplex states, as a function of interatomic distance $d$. In its ground state, the Ar dimer is weakly bound. Van der Waals interaction leads to a binding energy of $0.011 \mathrm{eV}$ [35]. Within our DFT-LDA ground state a slightly larger value of $0.04 \mathrm{eV}$ results, at an equilibrium distance of $d=3.4 \AA$ (in comparison with reported experimental values of $d=3.76 \AA$ [35] or $d=3.81 \AA$ [39]). In the excited state, on the other hand, the aforementioned depopulation of the antibonding HOMO and the population of the bonding LUMO generate

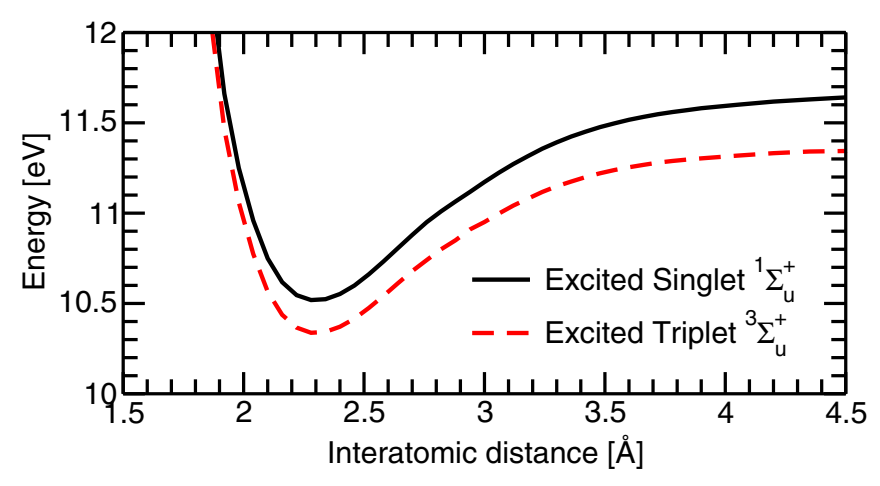

FIG. 4. Excited-state total energy of $\mathrm{Ar}_{2}$ as a function of the interatomic distance, relative to the ground-state total-energy minimum (at $0 \mathrm{eV}$ ). The excited state is given by the lowest-energy spin-triplet and spin-singlet excitation (mostly HOMO $\rightarrow$ LUMO), and its respective total energy results from adding the excitation energy to the DFT-LDA ground-state energy at each interatomic distance. Both for the singlet and the triplet state the equilibrium is at $d=2.3 \AA$ with minima $10.5 \mathrm{eV}(10.3 \mathrm{eV})$ above the ground state.

a "covalent" bond of $1.00 \mathrm{eV}(1.14 \mathrm{eV})$ binding energy in the triplet (singlet) excited state, with a bond length of $d=2.30 \AA$ in both cases. The binding energy is given by the difference between the energy minimum and the asymptotic limit at large distance $(11.33 \mathrm{eV}$ for the triplet, $11.65 \mathrm{eV}$ for the singlet state). The bond in each excited state is significantly shorter (by $1.1 \AA$ ) than the equilibrium distance of the ground state. Note that slightly different results are obtained when starting with DFT-PBE: The ground-state binding is negligible, and the excited-state binding energy amounts to $0.70 \mathrm{eV}(0.88 \mathrm{eV})$ for the spin-triplet (spin-singlet) excited state, at a bond length of $d=2.34 \AA$. The difference between the data based on LDA and PBE mostly result from differences in the ground-state PES, which (due to the construction of excited-state energies as the sum of ground-state energy plus excitation energy) directly transfers to the excited-state PES. All these data, which reflect the well-known formation of an excimer (i.e., "covalently" bonded dimer as long as the excitation persists), are in good agreement with data from CI calculations, which yields binding energy of 0.550 and $0.596 \mathrm{eV}$ at a bond length of $d=2.48 \AA$ for the spin-triplet and spin-singlet configuration [40].

\section{Mixture of on-site and charge-transfer excitations for a van der Waals bound dimer}

A further analysis of excited-state bonding requires a quantitative understanding of the configuration of the electronic excitation.

We start this discussion with the schematic diagram of Fig. 1(d). From the highest occupied $(o)$ and lowest unoccupied $(u)$ state on each component (A/B), the molecular orbitals of the complex are roughly given by

$$
\begin{gathered}
|\mathrm{HOMO}-1\rangle=\frac{1}{\sqrt{2}}\left(\left|o_{\mathrm{A}}\right\rangle+\left|o_{\mathrm{B}}\right\rangle\right), \\
|\mathrm{HOMO}\rangle=\frac{1}{\sqrt{2}}\left(\left|o_{\mathrm{A}}\right\rangle-\left|o_{\mathrm{B}}\right\rangle\right),
\end{gathered}
$$




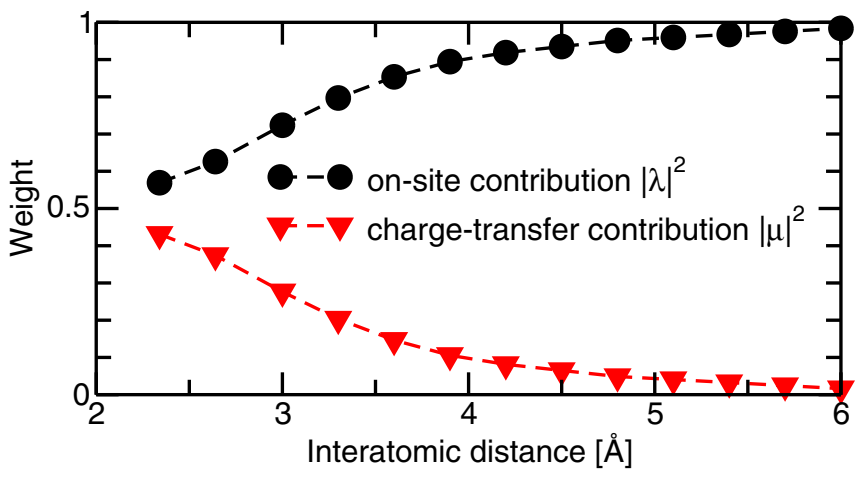

FIG. 5. Composition of the singlet excitation of $\mathrm{Ar}_{2}$ from onsite configurations on each atom $\mathrm{A} / \mathrm{B}\left(|\lambda|^{2}\right.$, upper curve) and chargetransfer configurations $\mathrm{A} \rightarrow \mathrm{B}$ and $\mathrm{B} \rightarrow \mathrm{A}\left(|\mu|^{2}\right.$, lower curve).

$$
\begin{gathered}
|\mathrm{LUMO}\rangle=\frac{1}{\sqrt{2}}\left(\left|u_{\mathrm{A}}\right\rangle+\left|u_{\mathrm{B}}\right\rangle\right), \\
|\mathrm{LUMO}+1\rangle=\frac{1}{\sqrt{2}}\left(\left|u_{\mathrm{A}}\right\rangle-\left|u_{\mathrm{B}}\right\rangle\right),
\end{gathered}
$$

without caring about renormalization of the prefactor $1 / \sqrt{2}$ due to spatial overlap of the orbitals on A and B. Note that depending on the arbitrary sign or phase in the definition of each original orbital, the signs inside each linear combination may be different from Eqs. (2)-(5). The HOMO-LUMO excitation would then be given by

$$
\begin{aligned}
|\mathrm{HOMO} \rightarrow \mathrm{LUMO}\rangle= & \frac{1}{2}\left(\left|o_{\mathrm{A}} \rightarrow u_{\mathrm{A}}\right\rangle-\left|o_{\mathrm{B}} \rightarrow u_{\mathrm{B}}\right\rangle\right. \\
& \left.+\left|o_{\mathrm{A}} \rightarrow u_{\mathrm{B}}\right\rangle-\left|o_{\mathrm{B}} \rightarrow u_{\mathrm{A}}\right\rangle\right),
\end{aligned}
$$

i.e., as a coherent, linear combination of four terms. In this simplified notation, details like Pauli's principle and the spin structure of the excitation are left out for clarity sake. The first two terms are on-site excitations within component A or $\mathrm{B}$ alone, while the last two terms constitute charge-transfer configurations with the hole on A and the electron on B, or vice versa. As one can see, the on-site and the chargetransfer configurations contribute with similar importance to the excitation.

Equation (6) turns out to be too simple (see the discussion further below), but it motivates the following general composition of the lowest-energy excitation $|S\rangle$ from on-site and charge-transfer contributions:

$$
\begin{aligned}
|S\rangle= & \lambda\left(\left|o_{\mathrm{A}} \rightarrow u_{\mathrm{A}}\right\rangle-\left|o_{\mathrm{B}} \rightarrow u_{\mathrm{B}}\right\rangle\right) \\
& +\mu\left(\left|o_{\mathrm{A}} \rightarrow u_{\mathrm{B}}\right\rangle-\left|o_{\mathrm{B}} \rightarrow u_{\mathrm{A}}\right\rangle\right)
\end{aligned}
$$

(neglecting the spin structure of the excited state, which can be either a triplet or a singlet state). This expression is symmetric in $\mathrm{A}$ and $\mathrm{B}$, which holds for complexes formed from two identical components A and B, e.g., the $\mathrm{Ar}_{2}$ dimer. Generalization to nonsymmetric cases would be straightforward.

We have analyzed our numerically determined BSE states in the notation of Eq. (7). Figure 5 shows the resulting weights $|\lambda|^{2}$ and $|\mu|^{2}$ which we observe for the case of the $\mathrm{Ar}_{2}$ dimer. At small interatomic distance, $|\lambda|^{2} \approx|\mu|^{2} \approx 1 / 2$, as suggested by Eq. (6). At larger interatomic distance, however, the charge-transfer contributions diminish and the on-site
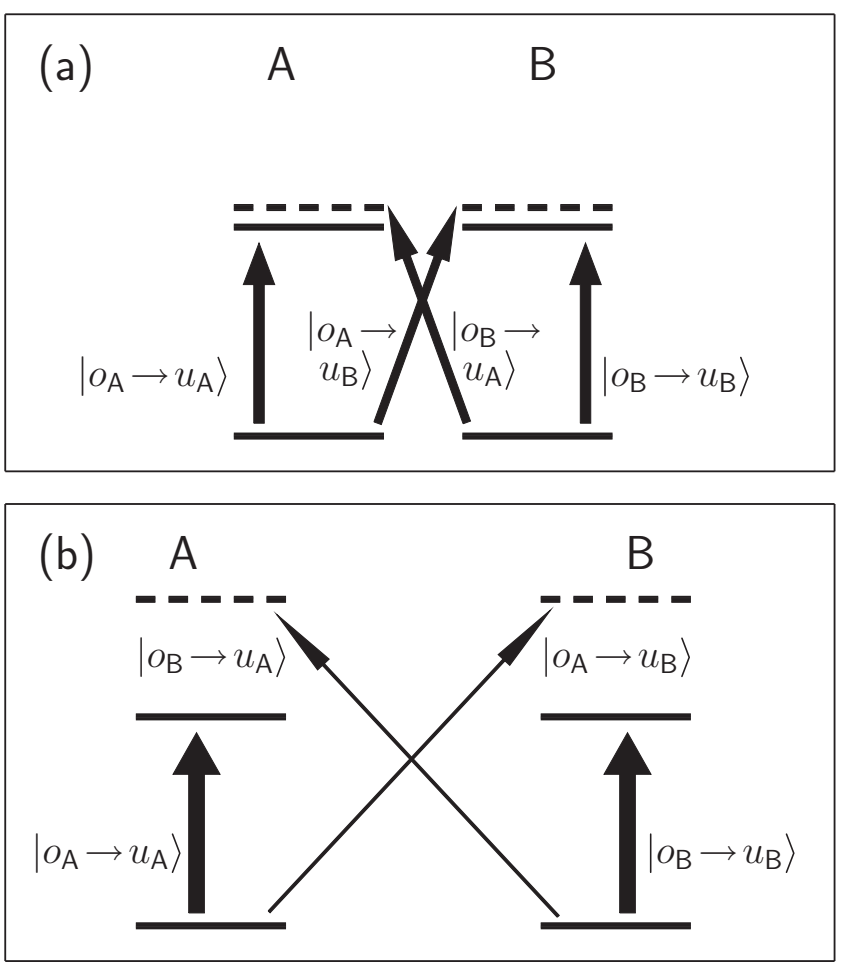

FIG. 6. Schematic illustration of the composition of an exciplex state from on-site configurations [vertical arrows, with prefactor $\lambda$ in Eq. (7)] and charge-transfer configurations [slanted arrows, with prefactor $\mu$ in Eq. (7)]. At small interatomic distance (a) on-site and charge-transfer configurations are of similar excitation energy, facilitating mixing and leading to prefactors $\lambda$ and $\mu$ of similar magnitude. At large interatomic distance (b) the charge-transfer configurations are of much higher energy (see text) and do not contribute significantly $(\mu \sim 0)$, as indicated by the thin slanted arrows.

contributions dominate, i.e., for two well-separated atoms charge transfer does not play a role. In between, the composition of the excitation depends drastically on the distance between the two components, showing a transition from an exciplex configuration at close distance to an on-site configuration at far distance.

One reason for the insignificance of the charge-transfer contributions at large distance (i.e., $\mu \rightarrow 0$ ) lies in their higher energy, as illustrated in Fig. 6. The on-site excitation within A has an energy of $\epsilon\left(u_{\mathrm{A}}\right)-\epsilon\left(o_{\mathrm{A}}\right)-W_{\mathrm{eh}}$, with $\epsilon$ denoting the energy levels and $-W_{\text {eh }}<0$ being the (screened) Coulomb interaction between electron and hole within A. On the other hand, the charge-transfer excitation energy between $\mathrm{A}$ and B amounts to $\epsilon\left(u_{\mathrm{A}}\right)-\epsilon\left(o_{\mathrm{B}}\right)-e^{2} / d$, which, for sufficiently large distance $d$, is higher than the on-site excitation energy. Here we focus on a homogeneous dimer with $A$ and $B$ being of the same element, i.e., $\epsilon\left(u_{\mathrm{A}}\right)=\epsilon\left(u_{\mathrm{B}}\right)$ and $\epsilon\left(o_{\mathrm{A}}\right)=\epsilon\left(o_{\mathrm{B}}\right)$. The charge-transfer configuration becomes thus too high in energy to contribute significantly to the excitation at large distance $d$. In other words, exciplex configurations (mixtures of on-site and charge-transfer configurations) require that $W_{\mathrm{eh}} \sim e^{2} / d$, which is only given at sufficiently close distance $d$.

Figure 6 also offers an intuitive explanation for the totalenergy lowering of Fig. 6(a) (at close distance) as compared 
to Fig. 6(b) (at far distance). While the on-site excitation energies (vertical arrows) are independent of distance, the admixture of the charge-transfer configurations in Fig. 6(a) lowers the energy, as can be discussed in terms of secondorder perturbation theory, even if their energy is higher than that of the on-site terms.

Note that Eq. (6) and the schematic diagram of Fig. 1(d) alone are not sufficient to fully explain the distance dependence of $\lambda$ and $\mu$. According to Fig. 1(d), the HOMO-LUMO transition should have a structure like Eq. (6) at any distance. However, one has to keep in mind that at increasing distance, the energetic splitting between $\sigma$ and $\sigma^{*}$ decreases (among the occupied as well as among the unoccupied orbitals). Consequently, the four excitations |HOMO $-1 \rightarrow$ LUMO $\rangle, \mid \mathrm{HOMO}-1 \rightarrow$ LUMO +1$\rangle,|\mathrm{HOMO} \rightarrow \mathrm{LUMO}\rangle$, and $|\mathrm{HOMO} \rightarrow \mathrm{LUMO}+1\rangle$ come closer in energy and mix in such a way that the lowest excitation (i.e., the data toward the right-hand side of Figs. 4 and 5) mostly contains on-site configurations of $\mathrm{A}$ and $\mathrm{B}$ [i.e., $\mu \rightarrow 0$ in Eq. (7)].

We note that near the ground-state equilibrium of $d=$ $3.4 \AA$ the composition of the Ar-dimer exciplex is roughly given by $|\lambda|^{2} \sim 0.8$ and $|\mu|^{2} \sim 0.2$. As shown below, layered materials show similar compositions of their excitations from intralayer and interlayer contributions.

\section{Summary}

We close this section on molecules by summarizing the most important findings:

(i) Between strongly interacting (i.e., covalently bonded) components, excitation energies will decrease with increasing bond length, i.e., $\partial \Omega_{S} / \partial d<0$ and $\partial E_{\text {tot }}^{*} / \partial d<0$. The excitation weakens the bond and tends to elongate it.

(ii) Between weakly interacting (i.e., van der Waals-bound) components, excitation energies will decrease with decreasing bond length, i.e., $\partial \Omega_{S} / \partial d>0$ and $\partial E_{\text {tot }}^{*} / \partial d>0$. The excitation strengthens or even generates the bond and tends to shorten it.

(iii) For the latter situation (ii), a significant amount of charge-transfer configurations is observed in the electronic excitations. The precise amount depends on the distance between the components and diminishes as the components move away from each other.

In the next section we demonstrate that the same behavior holds for weakly interacting extended layers.

\section{LAYERED MATERIALS}

In this section we demonstrate that all effects discussed above for molecules are equally valid for layered systems, illustrating the close analogy between these system classes. In detail, we discuss the following issues:

(i) Excitations of layered materials are subject to reduction in excitation energy when the material is laterally stretched. This is analogous to the reduction of excitation energies of covalently bonded molecules when the bond is elongated.

(ii) When individual monolayers are stacked on top of each other to form multilayers or three-dimensional crystals, the excitations are no longer pure intralayer excitations (which would correspond to on-site excitations of one atom in a

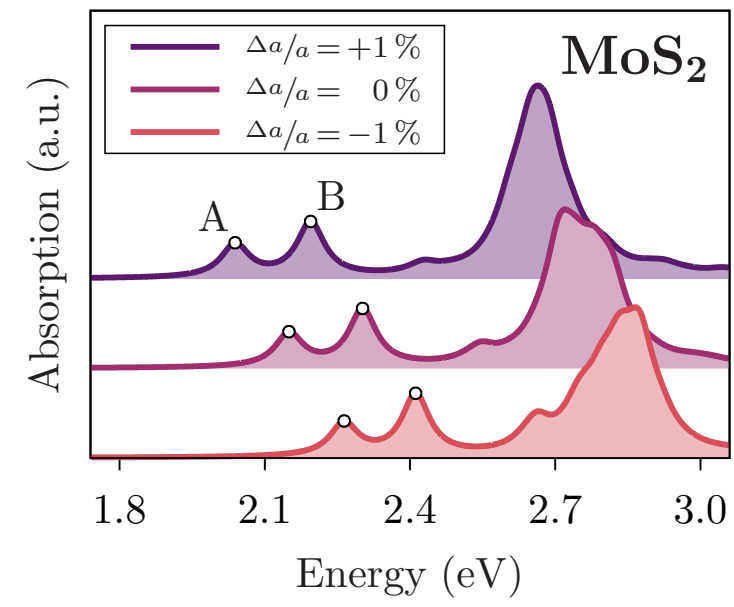

FIG. 7. Calculated optical spectrum of a monolayer of $\mathrm{MoS}_{2}$ at its equilibrium lattice constant $a_{0}$ and for modified lattice constants.

weakly bound dimer). Instead, they include a certain amount $(\sim 10 \%)$ of interlayer contributions with electron and hole on different layers (corresponding to the charge-transfer configurations in a weakly bound dimer of two noble-gas atoms) and should be regarded as exciplex states.

(iii) In analogy to an excimer, the exciplex states of layered materials observe a redshift, i.e., a reduction of their excitation energy when the material is compressed along the stacking direction.

Note that a redshift already occurs when monolayers are stacked into multilayer or bulk systems, due to the dielectric polarizability of the neighboring layers [26,41]. In addition to this effect, electronic hybridization and resulting admixture of charge-transfer configurations causes further redshift with a strong dependence on interlayer distance, as shown in Sec. IV B.

\section{A. Excitations of stretched layered materials}

A monolayer of a semiconducting 2D material (e.g., TMDC or $h$-BN) typically exhibits an optical spectrum as shown in Fig. 7, consisting of one or more exciton peaks that constitute the onset of the spectrum. For our discussion, these states play the same role as the excited states of the $\mathrm{H}_{2}$ molecule (cf. Fig. 2). It turns out that the excitation energy of these states also depends on the lattice constant of the monolayer. For illustration, Fig. 7 shows data for lattice constants changed by $\pm 1 \%$. Obviously, both the $A$ exciton and the $B$ exciton of a $\mathrm{MoS}_{2}$ monolayer are shifted to lower energy when the material is laterally stretched $[42,43]$. This effect is equivalent to the excitation-energy reduction in stretched $\mathrm{H}_{2}$. The bonds within a TMDC monolayer are of covalent nature. Generating an excitation depopulates bonding valence states and populates antibonding conduction states, as schematically illustrated in Fig. 1(b), equivalent to molecular physics, resulting in the same type of bond length (i.e., lattice-constant) dependence.

The dependence of excitation energies on the bond length or the lattice constant can be expressed by a deformation potential $[42,43]$, denoting the change in excitation energy 
TABLE I. Deformation potentials for selected exciton states in various materials, in $\mathrm{meV}$ per $1 \%$ of lateral lattice expansion $(\Delta a / a)$, calculated in $G W / \mathrm{BSE}\left(\mathrm{H}_{2}\right)$ and in $\mathrm{LDA}+G d W / \mathrm{BSE}$ (all twodimensional materials). The experimental data are from reversible application of biaxial strain [42] or of uniaxial strain [43]. In the latter case we assume that biaxial strain (i.e., lattice-constant increase) would simply yield a doubled effect, which we give here. In all cases the negative sign indicates decrease of excitation energy for increasing lattice constant.

\begin{tabular}{llc}
\hline \hline System & Theory $^{\mathrm{a}}$ & Expt. \\
\hline $\mathrm{MoS}_{2}$ monolayer, $A$ exciton & -110 & $-51^{\mathrm{b}}$ \\
$\mathrm{MoS}_{2}$ monolayer, $B$ exciton & -107 & $-49^{\mathrm{b}}$ \\
$\mathrm{MoSe}_{2}$ monolayer, $A$ exciton & -90 & $-33^{\mathrm{b}}$ \\
$\mathrm{MoSe}_{2}$ monolayer, $B$ exciton & -89 & $-30^{\mathrm{b}}$ \\
$\mathrm{WS}_{2}$ monolayer, $A$ exciton & -151 & $-94^{\mathrm{b}}$ \\
$\mathrm{WS}_{2}$ monolayer, $B$ exciton & -130 & \\
$\mathrm{WSe}_{2}$ monolayer, $A$ exciton & -134 & $-63^{\mathrm{b}},-108^{\mathrm{c}}$ \\
$\mathrm{WSe}_{2}$ monolayer, $B$ exciton & -111 & $-43^{\mathrm{b}},-100^{\mathrm{c}}$ \\
$h-\mathrm{BN}$ bulk, lowest exciton & -80 & \\
$\mathrm{Graphite}^{\mathrm{c}} \Omega=1.5 \mathrm{eV}$ & -35 & \\
$\mathrm{Graphite}$ at $\Omega=4.5 \mathrm{eV}$ & -80 & \\
$\mathrm{H}_{2}$ molecule, lowest triplet & -102 & \\
$\mathrm{H}_{2}$ molecule, lowest singlet & -58 & \\
\hline
\end{tabular}

aThis work.

${ }^{\mathrm{b}}$ Biaxial strain, Ref. [42].

${ }^{\mathrm{c}}$ Deduced from uniaxial strain, Ref. [43].

when the geometry is stretched by $1 \%$. Table I summarizes our calculated deformation potential for a number of covalently bonded systems. In addition to the TMDC monolayers we include two layered bulk systems, $h$-BN and graphite. Note that for graphite the dependence of excitation energies on the lattice constant requires particular analysis as graphite has no gap and its exciton states must be considered as resonant states (see the discussion in Sec. IV D). Table I also contains a number of experimental data from experiments in which a TMDC monolayer is mechanically stretched in one or two directions, after gluing the monolayer to a substrate which is then stretched $[42,43]$. Due to difficulties to transfer the substrate strain fully to the monolayer the measured data may be considered as lower bounds of the deformation potential,

TABLE II. Calculated intralayer and interlayer contributions to the lowest singlet excitons in bulk structures of two-dimensional semiconductors. All values are obtained at the experimental equilibrium distance of vertical stacking. Similarly to $h$-BN, the intralayer contribution becomes smaller at compression and larger at increased vertical stacking.

\begin{tabular}{lcc}
\hline \hline System & $\begin{array}{c}\text { Intralayer } \\
\text { contribution }\end{array}$ & $\begin{array}{c}\text { Interlayer } \\
\text { contributions }\end{array}$ \\
\hline $2 \mathrm{H}-\mathrm{MoS}_{2}$ & 0.89 & 0.11 \\
$2 \mathrm{H}-\mathrm{MoSe}_{2}$ & 0.92 & 0.08 \\
$2 \mathrm{H}-\mathrm{MoTe}_{2}$ & 0.90 & 0.10 \\
$2 \mathrm{H}-\mathrm{WS}$ & 0.87 & 0.13 \\
$2 \mathrm{H}-\mathrm{WSe}$ & 0.88 & 0.12 \\
$h-\mathrm{BN}$ & 0.73 & 0.27 \\
\hline \hline
\end{tabular}

which might in reality be larger than observed. In all systems studied here we observe deformation potentials of similar magnitude (about $-0.1 \mathrm{eV} / \%$ ), indicating that the underlying physical mechanisms and effects are similar: The excitation of a covalent bond turns a bonding into an antibonding configuration.

We note in passing that the manipulation of excitation energy by strain opens a path toward lateral localization of excitons in a TMDC monolayer, e.g., at positions where it is bent or otherwise deformed, accompanied by stretching [44]. Such localized states may be used for single-photon emission from specific locations at emission energies below the unstrained exciton absorption energy. On the other hand, the significant sensitivity of exciton energies to structure can cause inhomogeneous spectral broadening if the monolayer is not perfectly periodic, i.e., if it shows locally varying strain, e.g., due to lattice-constant variation on imperfect substrates.

\section{B. Mixture of intralayer and charge-transfer excitations for excitons of layered materials}

In this section we take a monolayer as one single quantummechanical object, integrating the details of its lateral intralayer physics into an orbital scheme as given by one object (e.g., A) in Fig. 1(c). Starting from this simplified picture, we now focus on the interaction between two (or more) such objects when they are brought in contact by stacking them vertically. Similarly to the case of $\mathrm{Ar}_{2}$, chemical inertness prevents strong chemical bonds between them and only allows for weak interaction, e.g., by van der Waals forces. Nonetheless, the orbitals can significantly overlap across the spatial gap between the layers, leading to hybridization as indicated in Fig. 1(c). A well-known consequence is the possibility of vertical band dispersion in bulk TMDC materials. Consequently, the same kind of exciplex states as in the case of $\mathrm{Ar}_{2}$ will be formed.

In the case of a single monolayer (e.g., of a TMDC) an exciton is composed of an electron and a hole within one layer. Such an intralayer excitation corresponds to the on-site excitation of, e.g., a noble-gas atom (cf. Sec. III B). In the case of two or more layers stacked on top of each other, excitons might naively be considered as being purely composed of such intralayer excitons. They would be delocalized, i.e., distributed over all layers. Their internal structure, however, would still be given by an intralayer excitation mechanism, i.e., the excited electron and hole would be located in the same layer as far as the internal spatial correlation is concerned.

In contrast, we find that charge-transfer configurations contribute significantly to the excitons. This can clearly be identified by analyzing the charge-density distribution of, e.g., the excited electron relative to the excited hole. As an example Fig. 8 displays the electron density of the $A$ exciton in bulk $2 \mathrm{H}-\mathrm{MoS}_{2}$. Note that we construct this $A$ exciton at the direct gap at the $K$ and $K^{\prime}$ point of the material's Brillouin zone (relevant for optical absorption), although smaller excitation energy can be found for indirect transitions in the $2 \mathrm{H}-\mathrm{MoS}_{2}$ bulk band structure (involving nonzero momentum of the exciton). The excited hole is located in the center of the plot. 


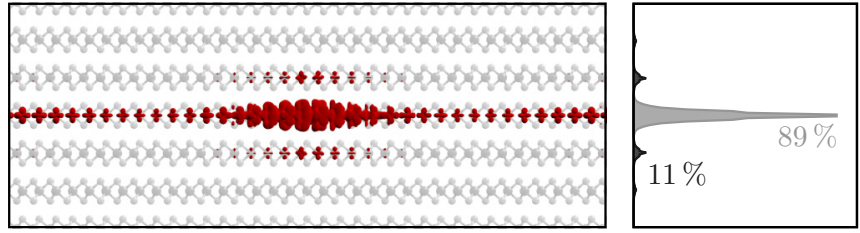

FIG. 8. Spatial density of the excited electron of the $A$ exciton in bulk $2 \mathrm{H}-\mathrm{MoS}_{2}$, as a side view of the crystal. The excited hole is located in the center of the plot. On the right-hand side the same quantity is shown after integration over the lateral coordinates. About $89 \%$ of the electron is located in the same layer as the hole (intralayer or on-site contribution), while $11 \%$ of the electron is located in other layers (interlayer or charge-transfer contribution).

Two-dimensional integration of the lateral degrees of freedom yields the one-dimensional line density profile of the electron shown on the right-hand side of Fig. 8. About $89 \%$ of the electron is located in the same layer as the hole, i.e., the $A$ exciton is dominated by intralayer configurations [corresponding to $|\lambda|^{2} \sim 0.89$ in Eq. (7)]. Nonetheless, interlayer configurations are not negligible; they contribute about $11 \%$ to the exciton [corresponding to $|\mu|^{2} \sim 0.11$ in Eq. (7)]. Data for other TMDC materials and for $h-\mathrm{BN}$ are compiled in Table II, showing a similar balance between intralayer and interlayer contributions in all materials investigated. Note that such interlayer contributions are still far away from dominant interlayer excitons observed at higher excitation energies (above the $A$ exciton), for which we find interlayer contributions of more than $50 \%$ [24].

Similarly to the weakly bound $\mathrm{Ar}_{2}$ dimer (see above), the balance between intralayer and interlayer contributions for layered materials depends sensitively on the interlayer distance (i.e., on the vertical lattice constant, $c$ ). As an example, we discuss the intralayer and interlayer contributions for the lowest vertical exciton in bulk $h$-BN. Compared to the TMDC materials, $h$-BN has much larger excitonic binding energy, and the exciton is easier to identify at all values of $c$. Due to these technical reasons we discuss all further effects for the case of bulk $h$-BN. Figure 9(a) shows relative to the hole the amplitude of the excited electron on the various layers for two values of the unit-cell height $c$ (i.e., interlayer distance $c / 2$, since $h$-BN has two layers per unit cell). The ground-state equilibrium vertical lattice constant amounts to $c_{0}=6.6 \AA$. At smaller perpendicular lattice constant $(c=6 \AA)$ the electron has an amplitude of $\sim 0.5$ on the layer of the hole, accompanied by significant amplitude on the neighboring layers, i.e., it is rather delocalized. At $c=7 \AA$, on the other hand, the electron is much more strongly localized on the same layer as the hole, with an amplitude of $\sim 0.85$.

The distance dependence of these effects results from the overlap of and hopping between the orbitals of adjacent layers. Both the overlap of the orbitals and thus the charge-transfer admixture depend exponentially on the interlayer distance, $c / 2$. This is demonstrated in Fig. 9(b), which displays the same data as in Fig. 9(a) as a function of $c$. The behavior of the black dots (intralayer contributions) is fully analogous to the on-site weight $|\lambda|^{2}$ for $\mathrm{Ar}_{2}$ (cf. Fig. 5). Similarly, the behavior of the interlayer contributions (e.g., the red triangles) resem-
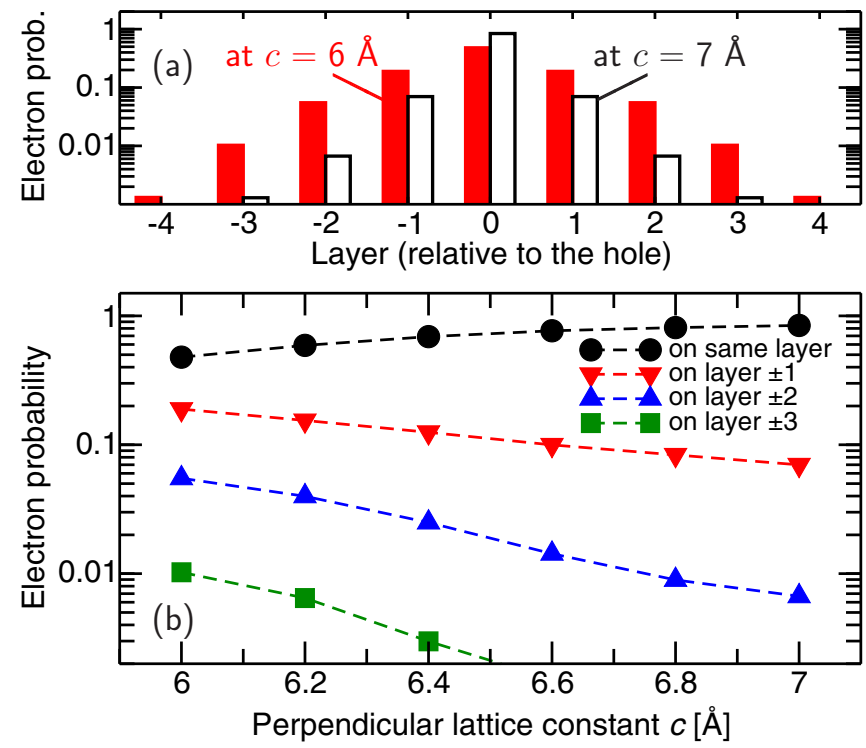

FIG. 9. Probability distribution of the excited electron relative to the hole for the lowest-energy exciton in bulk $h$-BN. (a) Shown as a function of distance from the layer on which the hole is located. (b) Same data as in (a) but shown as a function of perpendicular lattice constant $c$. The ground-state equilibrium is given by $c=6.6 \AA$.

bles the charge-transfer weight $|\mu|^{2}$ for $\mathrm{Ar}_{2}$. For increasing lattice constant $c$, the interlayer contributions would finally vanish (cf. $|\mu|^{2} \rightarrow 0$ for $d \rightarrow \infty$ in the case of $\mathrm{Ar}_{2}$ ).

\section{Interlayer-distance dependence of excitation energies}

In analogy to the excited-state energy of the molecular excimer $\mathrm{Ar}_{2}$, the (de-)population of occupied and empty bands on electronic excitation has significant consequences for the excited-state PES of layered systems. In particular, decrease of interlayer distance causes decrease of the excitation energy. This is shown for the case of $h$-BN by the lower curve of Fig. 10. This effect is now discussed in several steps.

First, the upper curve (open squares) reports calculations in which one of the two BN sheets has been removed from the unit cell. These results show the physics of a single layer of BN, calculated in supercells of 6-7 $\AA$ height. In principle, the optical excitation energies of a single layer should be independent of the supercell height in which it is described, provided that the layers are sufficiently far apart $(\gtrsim 5 \AA)$. For increasing supercell height both the fundamental $G W$ gap and the excitonic binding energy of $h$-BN should increase by the same amount, exactly compensating each other [27,45] (this does not hold when the layers are at touching distance, see below). In practice, however, the results from a numerical procedure might deviate from this exactly complementary behavior. In our case, the fundamental gap and the excitonic binding energy increase by 0.111 and $0.124 \mathrm{eV}$ when the supercell height increases from 6 to $7 \AA$, resulting in a small artificial exciton-energy drift of $0.013 \mathrm{eV}$. We believe that this stability of our data is sufficient to discuss the following issues. 


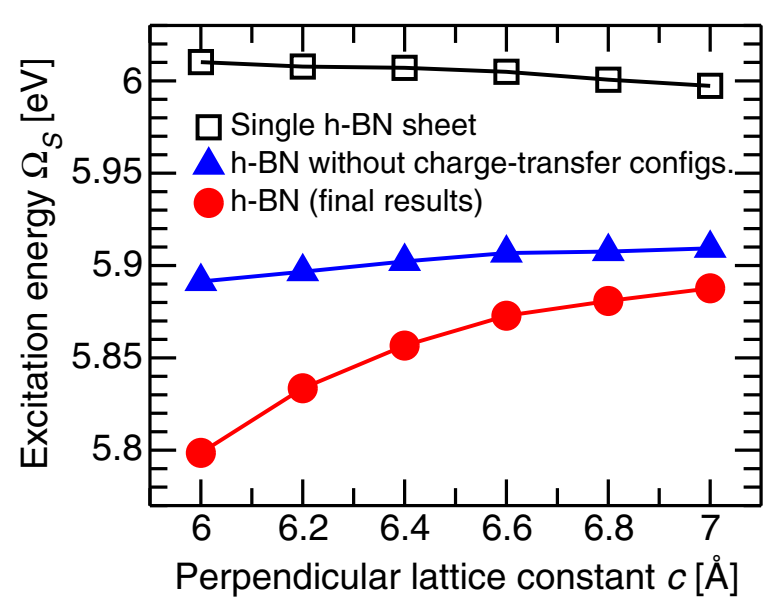

FIG. 10. Excitation energy of the lowest exciton of $h$-BN as a function of the perpendicular lattice constant $c$. Open squares refer to a single layer of $h$-BN, treated in the same unit cell as bulk $h$-BN (see text). Blue triangles refer to bulk $h$-BN with dielectricscreening interaction between the layers but no interlayer interaction of their electronic degrees of freedom (i.e., neglecting chargetransfer configurations between the layers, see text). Red circles denote the final BSE calculation for bulk $h-\mathrm{BN}$ (within LDA+GdW/ BSE).

Second, the excitons of bulk $h$-BN differ from those of a single layer due to interlayer polarizability: The polarizability of the other layers (now at touching distance) causes additional dielectric screening inside a layer. This effect reduces both the fundamental gap and the electron-hole interaction (see above) but now without complete cancellation because layers are much closer to each other and observe the nonhomogeneity (for distances $\lesssim 5 \AA$ ) of their dielectric polarizability $[41,45]$. In total, an effective redshift to lower energy by about $0.1 \mathrm{eV}$ results (see the blue triangles in Fig. 10). Such bathochromic effects are automatically included in the $G W / \mathrm{BSE}$ or LDA $+G d W / \mathrm{BSE}$ approach [45].

Third, in addition to the interlayer polarizability effect, quantum-mechanical hybridization of the hole states of neighboring layers (and of the electron states as well) can further lower the excitation energy. Such hybridization is automatically included in the $G W / \mathrm{BSE}$ or $\mathrm{LDA}+G d W / \mathrm{BSE}$ approach, but it can also be switched off (as had been done for the blue triangles in Fig. 10) for closer analysis. When the layers touch each other, the hybridization causes admixture of charge-transfer configurations (in which electron and hole are on different layers) to the intralayer exciton, i.e., formation of an exciplex state (see again Fig. 9).

Admixture of charge-transfer configurations lowers the excitation energy of the exciton, leading to the red dots in Fig. 10 as our final results. This redshift $(-0.09 \mathrm{eV}$ at $c=6 \AA$ compared to only $-0.02 \mathrm{eV}$ at $c=7 \AA$ ) is highly $c$ dependent, resulting from the exponential distance dependence discussed above. This particular effect is responsible for most of the $c$ dependence of the excitation energy, which amounts to $\partial \Omega / \partial c \approx 0.06 \mathrm{eV} / \AA$ at the experimental lattice constant of $c_{0}=6.6 \AA$.

For better comparison among the various systems, Table III lists the corresponding deformation potentials (meV
TABLE III. Calculated deformation potentials for selected exciton states in various materials in $\mathrm{meV}$ per $1 \%$ of perpendicular lattice expansion $(\Delta c / c)$. In all cases the positive sign indicates increase of excitation energy for increasing lattice constant. The values of the $\mathrm{Ar}_{2}$ dimer were taken at its DFT-LDA ground-state equilibrium bond length of $3.4 \AA$ (see Sec. III B).

\begin{tabular}{lcc}
\hline \hline System & State & $\begin{array}{c}\text { Deformation } \\
\text { potential }(\mathrm{meV} / \%)\end{array}$ \\
\hline bulk $h$-BN & Lowest exciton & +4 \\
Graphite & At $\Omega=1.5 \mathrm{eV}$ & +4 \\
Graphite & At $\Omega=4.5 \mathrm{eV}$ & +7 \\
$\mathrm{Ar}_{2}$ dimer & Lowest spin triplet & +14 \\
$\mathrm{Ar}_{2}$ dimer & Lowest spin singlet & +16 \\
\hline
\end{tabular}

per percentage of perpendicular lattice expansion), equivalent to those in Table I. Due to the exciplex nature of the excited states, these numbers are now all positive and are of similar magnitude to each other. Due to the more delicate nature of excitons in bulk TMDCs we do not discuss their deformation potentials here, which will be postponed to later work. Preliminary results indicate deformation potentials with the same sign as the data given in Table III but one order of magnitude smaller. Note the similarity between vertical strain of layered materials and dimer bond-length increase of $\mathrm{Ar}_{2}$, resulting from the similarity in the excited-state composition from on-site and charge-transfer contributions.

\section{Graphite}

The situation in graphite, while having a structure similar to that of $h-\mathrm{BN}$, is nonetheless significantly different in terms of its electronic structure and optical spectrum, which has no fundamental band gap. The spectrum can again fully be described by $G W /$ BSE [13-16,46,47], with self-energy effects and electron-hole attraction similar to semiconductors, as demonstrated by Yang et al. [16]. Note that due to the missing gap, the optical spectrum of graphite is dominated by excitonic resonances within a continuous spectrum instead of bound states. The spectrum thus does not exhibit sharp peaks but broad features, roughly following the density of states of the interband transitions. Nonetheless, it turns out that a conventional BSE calculation yields a spectrum in close resemblance with experimental data and that attractive electronhole correlation from the screened Coulomb interaction again plays a dominant role, analogously to bound excitons [16]. Within $\mathrm{LDA}+G d W / \mathrm{BSE}$ we obtain a spectrum (see Fig. 11) in good agreement with the $G W /$ BSE study by Yang et al., with a maximum at $4.30 \mathrm{eV}(4.50 \mathrm{eV}$ in $G W / \mathrm{BSE}$ [16]) which is $0.32 \mathrm{eV}$ lower than in the free interband spectrum $(0.27 \mathrm{eV}$ in $G W / \mathrm{BSE}$ [16]) due to electron-hole attraction. Our data are in reasonable agreement with typical experimental data [48,49] (see single symbols in Fig. 11). More data sets have been measured (see Ref. [50] for a recent review) but are not shown here for brevity sake. Note that the measured data shown in Fig. 11 exhibit some deviations between the two data sets, which might reflect difficulties in determining precise values of the dielectric function of graphite even today [50]. 


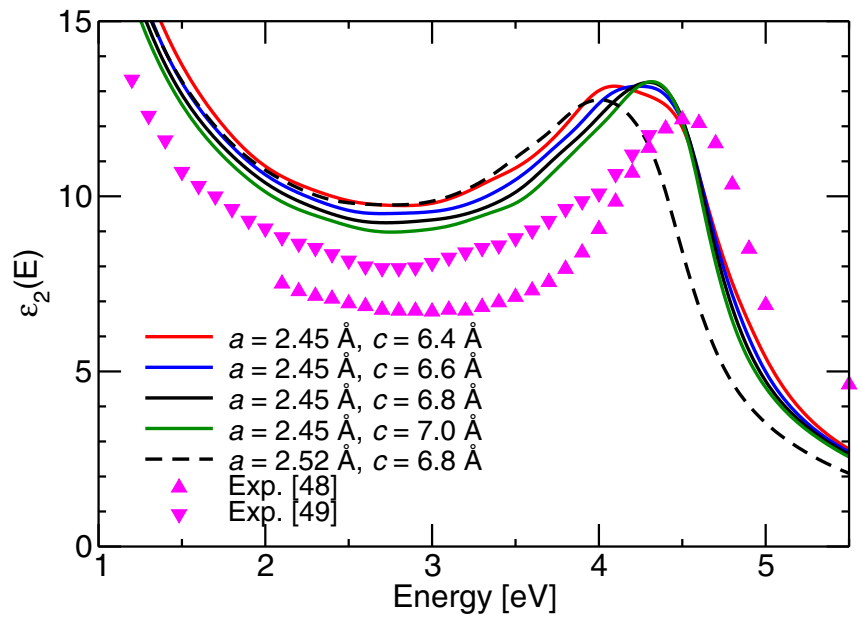

FIG. 11. Imaginary part of the dielectric function of graphite, calculated for various values for $a$ and $c$. Experimental data (for $a_{0}=2.45 \AA$ and $c_{0}=6.71 \AA$ ) are taken from Refs. [48] and [49] (see text).

Here we focus on the dependence of the spectrum and of the contributing resonant excitons on the lattice constants $a$ and $c$ around the experimental equilibrium of $a_{0}=2.45 \AA$ and $c_{0}=6.71 \AA$. Figure 11 shows the LDA $+G d W /$ BSE optical spectrum for various combinations of $a$ and $c$. For increasing $c$ the peak near 4.3-eV shifts to higher energies (by about $0.3 \mathrm{eV} / \AA$ ), which resembles the effect in $h$-BN (lower curve in Fig. 10) but seems to be even stronger. The situation in graphite is, however, more complex than in $h$-BN. The spectrum (including the peak at $4.3 \mathrm{eV}$ ) is formed from a large number of resonant rather than bound excitonic states. Changes in the spectrum not only result from changes in the excitation energy of each resonant exciton but also from changes in their optical dipole strength. Between 1 and $2 \mathrm{eV}$, for instance, the spectrum seems to be shifted toward lower energy for increasing $c$. As shown below, however, each exciton is rather shifted toward higher energy for increasing $c$.

In order to evaluate the energetic shift of individual excitons (comparable to Fig. 10) we consider the composition of each exciton $n$ from the interband transitions, i.e., $\left|\Psi_{S}\right\rangle=$ $\sum_{v c \mathbf{k}} A_{v c \mathbf{k}}^{(S)}|v c \mathbf{k}\rangle$ with $v / c$ denoting valence/conduction bands. Here we consider two occupied and two empty bands and 6400 k-points (cf. Sec. II). We assume that the composition of the interband transitions $|v c \mathbf{k}\rangle$ [see Eq. (1)] remains unchanged when the lattice constant is changed. The difference between the Hamilton operators $H^{\left[c_{1}\right]}$ and $H^{\left[c_{2}\right]}$ for two different lattice constants $c_{1}$ and $c_{2}$, i.e., $\Delta H=H_{v c \mathbf{k}, v^{\prime} c^{\prime} \mathbf{k}^{\prime}}^{\left[c_{1}\right]}$ $H_{v c \mathbf{k}, v^{\prime} c^{\prime} \mathbf{k}^{\prime}}^{\left[c_{2}\right]}$, can then be treated in lowest-order perturbation theory to evaluate the change of excitation energy for each exciton, $\Delta \Omega_{S}=\left\langle\Psi_{S}|\Delta H| \Psi_{S}\right\rangle$. To allow for a useful analysis under experimental conditions, these values are weighted with the optical dipole strength of each exciton, resulting in a continuous spectrum as shown in Fig. 12. This procedure is based on the idea that the excitation probability of each exciton (and therefore its relevance for the lattice-contraction mechanism) is proportional to its dipole strength and that each exciton, once excited, stays in its state without conversion

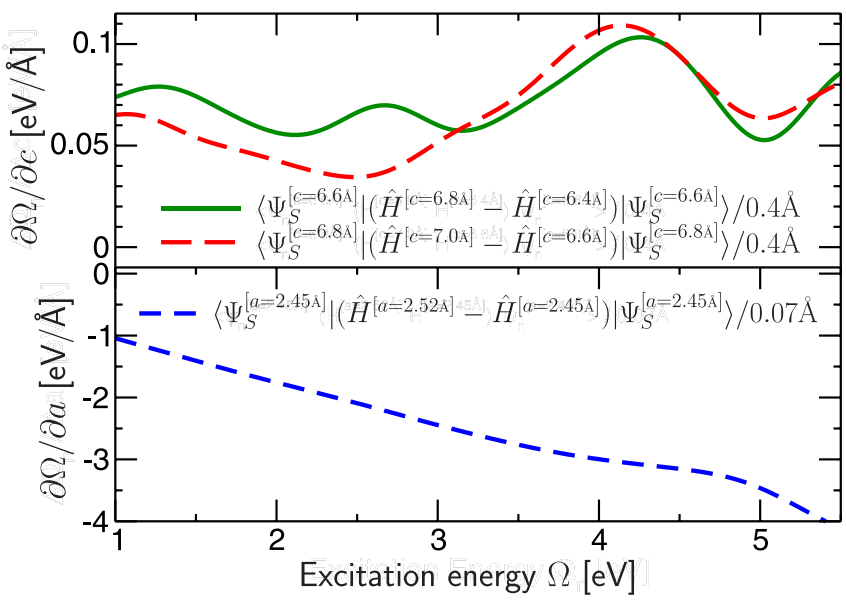

FIG. 12. Dependence of the excitation energy of graphite exciton states on the perpendicular lattice constant $c$ (upper panel) and on the lateral lattice constant $a$ (lower panel). Each exciton state is characterized by its excitation energy $\Omega_{S}$ (horizontal axis) and is weighted by its optical oscillator strength (quantifying its significance for the perpendicular lattice contraction studied in this paper), thus generating a spectrum. Note the different vertical scales in the two panels.

into other excitons. The entire procedure is quite delicate and approaches the numerical limits of our approach and the general limits of the approximations involved, as can be seen from the difference between the red and green curves in the upper panel of Fig. 12 (which were obtained at different lattice constant $c$ ). Note that effects from changing the lateral lattice constant $a$, relating to covalent bonds, are in general much more robust and thus simpler to evaluate than effects from changing the interplane distance. Nonetheless, $\partial \Omega / \partial c$ is clearly positive and of similar size as for $h$-BN. At $1.5 \mathrm{eV}$ (i.e., the excitation energy used in the experiment of Ref. [5]) we observe $\partial \Omega / \partial c \approx 0.06 \mathrm{eV} / \AA$, while at $4-4.5 \mathrm{eV}$ (i.e., at the peak in the optical spectrum), $\partial \Omega / \partial c \approx 0.1 \mathrm{eV} / \AA$ is slightly higher. The reason for the positive $\partial \Omega / \partial c$ is the same as for $h$-BN, i.e., at larger distance between the layers the charge-transfer configurations contribute less strongly to the excitons, and the excitation energy is raised.

For changes in the lateral lattice constant $a$, on the other hand, $\partial \Omega / \partial a$ is negative and one order of magnitude stronger (as for $h$-BN), as shown in the lower panel of Fig. 12. This corresponds to the significant negative energy shift of the peak in the optical spectrum (Fig. 11) by $-4.3 \mathrm{eV} / \AA$ when $a$ is increased. Note that this dependence on $a$ is numerically much more robust and simpler to evaluate than the dependence on $c$.

\section{E. Lateral expansion and vertical contraction of excited layered materials}

As discussed in Sec. III A electronic excitation of a covalent bond causes bond-length increase (e.g., in $\mathrm{H}_{2}$ ). In contrast, electronic excitation of a weak van der Waals bond causes bond contraction (e.g., in $\mathrm{Ar}_{2}$ ) due to formation of a "covalent" bond that did not exist before. In this section we show that these two mechanisms also exist in layered 
materials and that optical excitation can lead to lateral lattice expansion and to vertical lattice contraction simultaneously. We investigate this for the two cases of $h$-BN and graphite. In the case of graphite, perpendicular lattice contraction has in fact been observed experimentally [5].

One important difference to molecules concerns the excitation density. For a molecule, there is simply one excitation on one molecule, leading directly to the data as shown in Figs. 2 and 4. For an infinitely large periodic crystal, on the other hand, a single exciton would distribute over the entire sample, leading to vanishing effects on the total energy of the sample. Measurable effects can only be expected from high-density excitation with a macroscopic number of excitons.

The excitation energy $\Omega$ of an exciton adds to the total energy of the system, but this contribution depends on the number (or density) of excitons. With $E_{\text {tot }}^{(0)}$ being the groundstate energy per unit cell and $x$ being the density of excitons (states per unit cell), the excited total energy per unit cell amounts to

$$
E_{\mathrm{tot}}^{*}=E_{\mathrm{tot}}^{(0)}+x \Omega \text {. }
$$

We consider the behavior of $E_{\mathrm{tot}}^{*}$ as a function of $c$ in the vicinity of the ground-state equilibrium lattice constant $c_{0}$. Following elasticity theory [51,52], the ground-state total energy of bulk $h$-BN is given by a quadratic parabola

$$
E_{\mathrm{tot}}^{(0)}(c)=\frac{\sqrt{3} a_{0}^{2}}{4 c_{0}} C_{33}\left(c-c_{0}\right)^{2}
$$

(energy per unit cell, with the absolute minimum set to zero), with fixed lateral lattice constant $a_{0}$. The elastic constant $C_{33}$ is known experimentally; it amounts to $C_{33}=32.4 \mathrm{GPa}$ for $h$-BN [53,54]. The excitation energy, on the other hand, depends linearly on $c$, i.e., $\Omega(c) \approx \Omega\left(c_{0}\right)+(\partial \Omega / \partial c)\left(c-c_{0}\right)$ with $\partial \Omega / \partial c=0.06 \mathrm{eV} / \AA$ (see again Sec. IV C). This linear term (which has positive slope), when added to the parabola of $E_{\mathrm{tot}}^{(0)}(c)$, shifts the minimum of the parabola by $\Delta c<0$. The new equilibrium (i.e., $\partial E_{\mathrm{tot}}^{*} / \partial c=0$ ) is found at

$$
\Delta c=c-c_{0}=-\frac{2 c_{0}(\partial \Omega / \partial c) x}{\sqrt{3} a_{0}^{2} C_{33}} .
$$

Employing the above-given data, we obtain $\Delta c=-0.35 \AA \times x$. The resulting lattice contraction is shown in the lower panel of Fig. 13. Note that high exciton density $x$ is required to achieve a measurable effect.

In contrast to the perpendicular contraction of $c$, the lateral lattice constant $a$ is shifted in the opposite direction. In analogy to Eq. (9), elasticity theory yields, now as a function of $a$, a ground-state energy (per unit cell) of

$$
E_{\mathrm{tot}}^{(0)}(a)=\frac{\sqrt{3} c_{0}}{2}\left(C_{11}+C_{12}\right)\left(a-a_{0}\right)^{2}
$$

with fixed vertical lattice constant $c_{0}$. Analogously to the discussion above, the addition of the $a$-dependent excitation energy shifts the equilibrium lattice constant by

$$
\Delta a=a-a_{0}=-\frac{(\partial \Omega / \partial a) x}{\sqrt{3} c_{0}\left(C_{11}+C_{12}\right)} .
$$

Compared to the $c$ dependence, $\partial \Omega / \partial a=-3.2 \mathrm{eV} / \AA$ is much stronger, but the elastic constants for lateral deformation

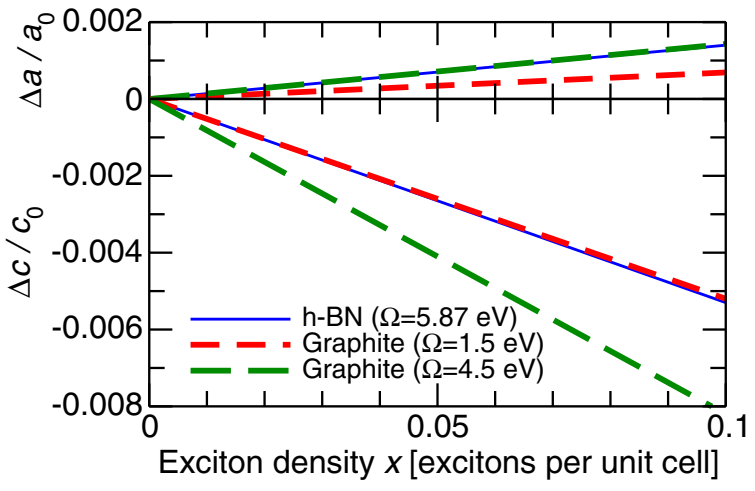

FIG. 13. Relative lateral lattice expansion (upper panel) and perpendicular lattice contraction (lower panel) of hexagonal boron nitride (when excited at its lowest-energy exciton at $5.87 \mathrm{eV}$ ) and of graphite (when excited by light of $1.5-$ and $4.5-\mathrm{eV}$ photon energy, respectively), as a function of exciton density $x$.

are much stronger, as well (with $C_{11}+C_{12}=1250 \mathrm{GPa}$ for $h$-BN [53,54]), yielding an increase of $a$ which is much weaker than the decrease of $c$ (see Fig. 13). Most importantly, $\partial \Omega / \partial a$ is negative because the excitons weaken the lateral covalent bonds by the "antibonding" configuration for which the interatomic attraction is reduced. Consequently, $\Delta a=$ $0.035 \AA \times x>0$ in contrast to $\Delta c<0$.

For graphite we observe analogous effects. Since there are no bound excitons in the material, we consider optical excitation at a given energy, which would generate resonant excitons at that energy. Assuming that these resonant excitons are sufficiently long lived, they would lead to analogous effects as discussed above for $h$-BN.

The deformation potentials for the resonant excitons in graphite were discussed in Sec. IV D. In combination with the elastic constants of $C_{33}=36.5 \mathrm{GPa}$ and $C_{11}+C_{12}=$ $1240 \mathrm{GPa}$ [55], we arrive at lateral lattice expansion by $\Delta a=$ $0.017 \AA \times x$ at $\Omega=1.5 \mathrm{eV}(0.035 \AA \times x$ at $\Omega=4.5 \mathrm{eV})$ and perpendicular contraction by $\Delta c=-0.35 \AA \times x$ at $\Omega=$ $1.5 \mathrm{eV}(-0.55 \AA \times x$ at $\Omega=4.5 \mathrm{eV})$. The resulting data for relative expansion and contraction (see Fig. 13) closely resemble those of $h$-BN but with a minor dependence on the photon energy employed. All conclusions for $h$-BN are equally valid for graphite.

Perpendicular lattice contraction of graphite was observed experimentally as a result of laser excitation at an energy of $1.5 \mathrm{eV}$ [5]. The measured data indicate contraction of up to $0.05 \%$ [5]. According to our theory, this would indicate an exciton density of about 0.01 , i.e., about one exciton per 100 unit cells. Note that this should not be confused with the internal size of a single exciton ("exciton radius"), which is of no relevance for the present discussion.

\section{CONCLUSIONS}

In conclusion, we have shown that in weakly bound condensed-matter systems electronic excitations have a special nature and the systems should be regarded as exciplex complexes. These exciplex states are composed from on-site and charge-transfer configurations, with the latter dominat- 
ing at close distance between the molecular components or between the layers, respectively. This distance dependence of the state composition transfers into an attractive distance dependence of their excitation energy, i.e., their excitation energy decreases when the interatomic distances are reduced. In turn, this means that a covalent-like bond or a perpendicular lattice contraction can be generated which only exists as long as the excitation is present. This is in contrast to the excitation physics of covalent bonds, in which excitation energies decrease when the interatomic distances are enlarged. We have evaluated all these issues by ab initio many-body perturbation theory, notably the $G W$ approximation or its simplified
LDA $+G d W$ version, both combined with the Bethe-Salpeter equation.

\section{ACKNOWLEDGMENTS}

This work has been supported by the German Research Foundation (Deutsche Forschungsgemeinschaft) in terms of the Collaborative Research Centre SFB 1083. T.D. acknowledges financial support from the Villum foundation. We gratefully acknowledge the computing time granted by the John von Neumann Institute for Computing and provided on the supercomputer JUWELS at Jülich Supercomputing Centre.
[1] J. B. Birks, Rep. Prog. Phys. 38, 903 (1975).

[2] Y. Wang, O. Haze, J. P. Dinnocenzo, S. Farid, R. Farid, and I. R. Gould, J. Org. Chem. 72, 6970 (2007).

[3] J. Liang, Q. L. Nguyen, and S. Matsika, Photochem. Photobiol. Sci. 12, 1387 (2013).

[4] J. Kalinowski, M. Cocchi, D. Virgili, V. Fattori, and J. A. G. Williams, Adv. Mater. 19, 4000 (2007).

[5] F. Carbone, P. Baum, P. Rudolf, and A. H. Zewail, Phys. Rev. Lett. 100, 035501 (2008).

[6] B. Arnaud, S. Lebegue, P. Rabiller, and M. Alouani, Phys. Rev. Lett. 96, 026402 (2006).

[7] G. Cappellini, V. Fiorentini, K. Tenelsen, and F. Bechstedt, in Gallium Nitride and Related Materials, edited by R. D. Dupuis, J. A. Edmond, F. A. Ponce, and S. Nakamura, MRS Symposia Proceedings No. 395 (Materials Research Society, Pittsburgh, 1996).

[8] G. Cappellini, G. Satta, M. Palummo, and G. Onida, Phys. Rev. B 64, 035104 (2001).

[9] C. Attaccalite, M. Bockstedte, A. Marini, A. Rubio, and L. Wirtz, Phys. Rev. B 83, 144115 (2011).

[10] J. Koskelo, G. Fugallo, M. Hakala, M. Gatti, F. Sottile, and P. Cudazzo, Phys. Rev. B 95, 035125 (2017).

[11] R. Mamy, J. Thomas, G. Jezequel, and J. C. Lemonnier, J. Phys. Lett. 42, 473 (1981).

[12] K. Watanabe, T. Tanaguchi, and H. Kanda, Nat. Mater. 3, 404 (2004).

[13] A. Grüneis, C. Attaccalite, T. Pichler, V. Zabolotnyy, H. Shiozawa, S. L. Molodtsov, D. Inosov, A. Koitzsch, M. Knupfer, J. Schiessling, R. Follath, R. Weber, P. Rudolf, L. Wirtz, and A. Rubio, Phys. Rev. Lett. 100, 037601 (2008).

[14] A. Grüneis, C. Attaccalite, L. Wirtz, H. Shiozawa, R. Saito, T. Pichler, and A. Rubio, Phys. Rev. B 78, 205425 (2008).

[15] P. E. Trevisanutto, C. Giorgetti, L. Reining, M. Ladisa, and V. Olevano, Phys. Rev. Lett. 101, 226405 (2008).

[16] L. Yang, J. Deslippe, C.-H. Park, M. L. Cohen, and S. G. Louie, Phys. Rev. Lett. 103, 186802 (2009).

[17] J. Yan, K. W. Jacobsen, and K. S. Thygesen, Phys. Rev. B 86, 045208 (2012).

[18] G. Onida, L. Reining, and A. Rubio, Rev. Mod. Phys. 74, 601 (2002).

[19] M. Rohlfing and S. G. Louie, Phys. Rev. B 62, 4927 (2000).

[20] M. Rohlfing, Phys. Rev. B 82, 205127 (2010).

[21] E. Artacho, M. Rohlfing, M. Cote, P. D. Haynes, R. J. Needs, and C. Molteni, Phys. Rev. Lett. 93, 116401 (2004).
[22] M. S. Kaczmarski, Y. Ma, and M. Rohlfing, Phys. Rev. B 81, 115433 (2010).

[23] B. Baumeier, M. Rohlfing, and D. Andrienko, J. Chem. Theory Comput. 10, 3104 (2014).

[24] A. Arora, M. Drüppel, R. Schmidt, T. Deilmann, M. R. Molas, P. Marauhn, S. Michaelis de Vasconcellos, M. Potemski, M. Rohlfing, and R. Bratschitsch, Nat. Commun. 8, 639 (2017).

[25] A. Arora, T. Deilmann, P. Marauhn, M. Drüppel, R. Schneider, M. R. Molas, D. Vaclavkova, S. Michaelis de Vasconcellos, M. Rohlfing, M. Potemski, and R. Bratschitsch, Nanoscale 10, 15571 (2018).

[26] Y. Niu, S. Gonzalez-Abad, R. Frisenda, P. Marauhn, M. Drüppel, P. Gant, R. Schmidt, N. S. Taghavi, D. Barcons, A. J. Molina-Mendoza, S. Michaelis de Vasconcellos, R. Bratschitsch, D. Perez De Lara, M. Rohlfing, and A. Castellanos-Gomez, Nanomaterials 8, 725 (2018).

[27] M. Drüppel, T. Deilmann, J. Noky, P. Marauhn, P. Krüger, and M. Rohlfing, Phys. Rev. B 98, 155433 (2018).

[28] S. Ismail-Beigi and S. G. Louie, Phys. Rev. Lett. 90, 076401 (2003).

[29] Y. Ma and M. Rohlfing, Phys. Rev. B 77, 115118 (2008).

[30] Y. Ma, M. Rohlfing, and A. Gali, Phys. Rev. B 81, 041204 (2010).

[31] J. Harl, L. Schimka, and G. Kresse, Phys. Rev. B 81, 115126 (2010).

[32] S. Grimme, J. Comput. Chem. 27, 1787 (2006).

[33] A. Tkatchenko, R. A. DiStasio, R. Car, and M. Scheffler, Phys. Rev. Lett. 108, 236402 (2012).

[34] O. Gunnarson and P. Johansson, Int. J. Quantum Chem. 10, 307 (1976).

[35] G. Herzberg, Molecular Spectra and Molecular Structure, Vol. III: Electronic Spectra and Electronic Structure of Polyatomic Molecules (Van Nostrand, New York, 1966).

[36] W. Kolos and L. Wolniewicz, J. Chem. Phys. 43, 2429 (1965).

[37] L. Wolniewicz and K. Dressler, J. Chem. Phys. 88, 3861 (1988).

[38] L. Wolniewicz, J. Chem. Phys. 103, 1792 (1995).

[39] Y. Tanaka and K. Yoshino, J. Chem. Phys. 53, 2012 (1970).

[40] J. Yates, W. Ermler, N. Winter, P. Christiansen, Y. Lees, and K. Pitzner, J. Chem. Phys. 79, 6145 (1983).

[41] M. Drüppel, T. Deilmann, P. Krüger, and M. Rohlfing, Nat. Commun. 8, 2117 (2017).

[42] R. Frisenda, M. Drüppel, R. Schmidt, S. Michaelis de Vasconcellos, D. Perez de Lara, R. Bratschitsch, M. Rohlfing, and A. Castellanos-Gomez, 2D Mater. Appl. 1, 10 (2017). 
[43] R. Schmidt, I. Niehues, R. Schneider, M. Drüppel, T. Deilmann, M. Rohlfing, S. Michaelis de Vasconcellos, A. Castellanos-Gomez, and R. Bratschitsch, 2D Mater. 3, 021011 (2016).

[44] J. Kern, I. Niehues, P. Tonndorf, R. Schmidt, D. Wigger, R. Schneider, T. Stiehm, S. M. de Vasconcellos, D. E. Reiter, T. Kuhn, and R. Bratschitsch, Adv. Mater. 28, 7101 (2016).

[45] M. Rohlfing, Phys. Rev. Lett. 108, 087402 (2012).

[46] V. N. Strocov, P. Blaha, H. I. Starnberg, M. Rohlfing, R. Claessen, J.-M. Debever, and J.-M. Themlin, Phys. Rev. B 61, 4994 (2000).

[47] V. N. Strocov, A. Charrier, J.-M. Themlin, M. Rohlfing, R. Claessen, N. Barrett, J. Avila, J. Sanchez, and M.-C. Asensio, Phys. Rev. B 64, 075105 (2001).
[48] D. L. Greenaway, G. Harbeke, F. Bassani, and E. Tosatti, Phys. Rev. 178, 1340 (1969).

[49] A. B. Kuzmenko, E. van Heumen, F. Carbone, and D. van der Marel, Phys. Rev. Lett. 100, 117401 (2008).

[50] B. T. Draine, Astrophys. J. 831, 109 (2016).

[51] Due to difficulties of DFT-LDA in describing the weak interlayer interaction in layered materials, we refrain from using it for the elasticity.

[52] See, e.g., M. Born and K. Huang, Dynamical Theory of Crystal Lattices (Clarendon Press, Oxford, 1954), Chap. 11.

[53] J. F. Green, T. K. Bolland, and J. W. Bolland, J. Chem. Phys. 64, 656 (1976).

[54] R. Ramani, K. K. Mani, and R. P. Singh, Phys. Status Solidi B 86, 759 (1978).

[55] O. L. Blakslee, D. G. Proctor, E. J. Seldin, G. B. Spence, and T. Weng, J. Appl. Phys. 41, 3373 (1970). 